\title{
Richter über Leben und Tod?
}

Die praktische Umsetzung von Wünschen auf Behandlungsabbruch mit lebensbeendender Zielsetzung

Der Wunsch, die Behandlung eines unheilbar Erkrankten abbrechen zu dürfen, um ihn »in Frieden sterben zu lassen «, wird und wurde immer wieder an den Betreuungsrichter $^{1}$ herangetragen, auch vor Erlass der grundlegenden Entscheidungen des OLG Frankfurt vom 15.07.1998 ${ }^{2}$ und des BGH vom 17.03.2003. ${ }^{3}$ Dabei liegt dem Betreuungsrichter allerdings bislang nur selten eine Patientenverfügung vor, in der der Patient eigene Anordnungen für den Fall getroffen hat, dass er selbst keine Entscheidungen mehr zu treffen imstande ist. Patientenverfügungen werden inzwischen zwar häufig erstellt, ihre Verfasser sind aber von der »letzten Phase ihres Lebens« offenbar noch ein Stück weit entfernt.

\section{Fallbeispiele}

Der Wunsch auf Behandlungsabbruch oder, wie es besser formuliert werden sollte, der Wunsch auf Umstellung des Behandlungsziels von Lebensverlängerung und -erhaltung auf Schmerz und Beschwerden lindernde medizinische und pflegerische Maßnahmen $^{4}$, kommt also in der Praxis meist von Angehörigen, von Betreuern, von Ärzten oder von Pflegeheimen, aber nur vereinzelt vom Patienten selbst oder auch nur von dessen sicher belegten mutmaßlichem Willen.

Gegen den Begriff »Behandlungsabbruch« wenden sich aus ärztlicher Sicht auch Borasio/Putz/Eisenmenger. ${ }^{5}$ Eine palliativmedizinische Behandlung am Lebensende stelle weder eine Minimaltherapie, noch gar einen Therapieabbruch dar, sondern die Fortführung der für diesen Patienten optimalen Therapie mit geändertem Therapieziel. Die Lebensverlängerung werde dabei durch die Maximierung der Lebensqualität als Therapieziel abgelöst. Bei einem bewusstlosen Patienten, liege nun eine Patientenverfügung vor oder nicht, seien lebensverlängernde Maßnahmen (zum Beispiel Intubation und Beatmung bei respiratorischer Insuffizienz, PEG bei Schluckunfähigkeit, aber auch die Gabe von Antibiotika bei Pneumonie) nicht schon deswegen »per se « indiziert, weil sie mit Wahrscheinlichkeit das biologische Leben des Patienten verlängerten. Die Auswahl der im Einzelfall richtigen von im allgemeinen mehreren The-

1 Vom Gesetz ungeachtet der Abschaffung der Vormundschaft für Erwachsene auch innerhalb des Betreuungsrechts weiterhin als Vormundschaftsrichter bezeichnet.

2 NJW 1998, 2747.

3 NJW 2003, 1588.

4 So die Grundsätze der Bundesärztekammer zur ärztlichen Sterbebegleitung Abschnitt II, veröffentlicht in Deutsches Ärzteblatt Jg. 101 Heft 19 vom 7. Mai 2004, A 1298.

5 In einem Aufsatz zu dem Beschluss des BGH in Deutsches Ärzteblatt Jg. 100 Heft $31-32$, vom 4. August 2003, A 2062. 
rapieoptionen müsse tunlichst im Konsens mit den engsten Familienmitgliedern/ Bezugspersonen erfolgen. Die Patientenverfügung sei dabei dann verbindliche Grundlage, wenn sie sich eindeutig auf die aktuelle Situation des Patienten beziehen lasse. Könne ein Konsens nicht erzielt werden, stelle eine vormundschaftsgerichtliche Prüfung einen guten Weg dar, um aus der Sicht einer dritten externen [und unabhängigen! d. Verf.] Instanz die Gründe für den Entscheidungskonflikt zu klären und diesen nach Möglichkeit aufzulösen. ${ }^{6}$

\section{a) Fallbeispiele bei von den Angehörigen ausgehendem Abbruchwunsch}

\section{Fall 1:}

Bei einer nicht mehr einwilligungsfähigen Patientin soll eine PEG-Sonde gelegt werden. Noch vor Durchführung der richterlichen Anhörung ruft eine Enkelin bei dem Betreuungsrichter an, stellt sich als Internistin vor und drängt darauf, das Legen der PEG-Sonde zu unterlassen, »um den Sterbeprozess nicht aufzuhalten «. Die Betroffene sei eine sehr selbstbewusste Geschäftsfrau gewesen, die niemals derart pflegebedürftig hätte weiterleben wollen, wie es jetzt der Fall sei. Bei der Anhörung wird die Betroffene zwar einwilligungsunfähig, aber mit wachen Augen vorgefunden. Nach Auskunft der Mitarbeiter des Pflegeheims hört sie noch mit sichtlichem Vergnügen Radio, Hinweise auf starken Leidensdruck oder Todessehnsucht liegen nicht vor. Die PEG-Sonde wurde gelegt. ${ }^{7}$

Die bloße Annahme (!) der Enkelin, die Großmutter würde nie in einem solcherart pflegebedürftigen Zustand weitergelebt haben wollen, stellt sich als reine Mutmaßung einer Angehörigen, nicht aber als konkretes Indiz auf den mutmaßlichen Willen der Patientin selbst dar. In dem sogenannten »Kemptener Fall $\aleph^{8}$ war es um eine Patientin gegangen, die acht bis zehn Jahren zuvor gelegentlich eines im Fernsehen geschilderten Pflegefalls mit Gliederversteifung und Wundliegen geäußert hatte, »so《 wolle sie nicht enden. Diese später nicht wiederholte Äußerung, so der BGH, biete keine tragfähige Grundlage für eine mutmaßliche Einwilligung zum Behandlungsabbruch. Sie könne einer momentanen Stimmung entsprungen sein. Ihre eigene Situation an die zehn Jahre später habe die Patientin nicht vorausahnen oder abschätzen können.

\section{Fall 2:}

Die Betreuerin wollte bei ihrer Schwester eine anstehende Sanierung des Herzschrittmachers nicht mehr vornehmen lassen, um dieser angesichts ihres ausgeprägten dementiellen Zustands ein natürliches Sterben zu ermöglichen. Das Gericht lehnte dieses Vorgehen ab.

Aus den Gründen: Bei der richterlichen Anhörung der Betroffenen zeigte sich ein komplettes Demenzsyndrom. Die Betroffene lief aber herum und versuchte, in einer ihrem dementiellen Zustand entsprechenden Art, den Richter zu begrüßen. Einen schwer leidenden Eindruck machte sie in keiner Weise. Seitens des Pflegepersonals wurde berichtet, sie laufe in dieser Weise immer herum. Manchmal komme sie zu

6 Dies gelingt in der Praxis in der ganz überwiegenden Zahl der Fälle.

7 Fallbeispiel aus Seichter, Einführung in das Betreuungsrecht, 2. Auflage, S. 138.

8 NJW 1995, 204. 
Sturz, was möglicherweise mit Funktionsstörungen des Herzschrittmachers zusammenhänge. Damit zeigt die Betroffene, ungeachtet ihrer Demenz, anders als etwa Patienten im Dauerkoma, etwa mit apallischem Syndrom, noch eine Vielzahl eigener Lebensäußerungen. Die Voraussetzungen, unter denen nach neuerer Auffassung lebenserhaltende Maßnahmen abgebrochen werden dürfen, liegen damit auch nicht ansatzweise vor. ${ }^{9}$

An diesem Fall wird die Problematik einer in Patientenverfügungen häufig anzutreffenden Wendungen deutlich, lebenserhaltende Maßnahmen sollten unterbleiben, wenn ein »menschenwürdiges Dasein « nicht mehr möglich sei. ${ }^{10}$ Diese Patientin führte in ihrer Demenz ein durchaus menschenwürdiges Leben! Wollte man hier eine Beendigung lebenserhaltender und -verlängernder Maßnahmen zulassen (oder gar fordern!), wäre keinerlei Grenzziehung mehr möglich zur Vernichtung lebensunwerten Lebens, wie es im nationalsozialistischen Deutschland praktiziert wurde.

Fall 3:

Der Hausarzt bittet das Gericht um Hilfe. Die Angehörigen seiner 104(!) Jahre alten Patientin meinten, bei dem Alter müsse man sie jetzt doch sterben lassen. Er, der Arzt, habe erhebliche Bedenken gegen einen Behandlungsabbruch.

Bei der richterlichen Anhörung reagierte die Betroffene auf ihren Namen und sprach auch. Wirkliche Dialogfähigkeit war jedoch zweifelhaft. Die Betroffene wirkte den Umständen entsprechend fröhlich, Todessehnsucht lag nach dem Eindruck des Richters, den die pflegenden Angehörigen bestätigten, nicht vor. Eigentliches Fallproblem war, dass die in die Pflege stark eingebundene Schwiegertochter, entsprechend dem Alter der Betroffenen selbst schon sehr betagt und zudem an Krebs erkrankt, erschöpft sei und zumindest eine Pflegepause brauche. Einzige Lösung sei eine Verlegung der Betroffenen in ein Heim, was diese aber ablehne. Das Gericht ordnete die Verbringung der Betroffenen in ein Heim für Kurzzeitpflege $a^{11}$ und setzte zugleich eine außenstehende Betreuerin ein. Diese berichtete nach einem Heimbesuch, die Betroffene habe mittlerweile eingesehen, dass es anders nicht gegangen sei. Sie erhalte in dem im gleichen Dorf gelegenen Pflegeheim reichlich Besuch von Freunden und Nachbarn. Knapp einen Monat später verstarb die Betroffene im Altenheim, wenige Wochen darauf erlag ihre Schwiegertochter, die sie ungeachtet ihres eigenen Leidens jahrelang aufopferungsvoll gepflegt hatte, ihrer Krebserkrankung. ${ }^{12}$

An diesem Fall wird deutlich, dass man die Angehörigen, die für ihre Angehörige ja Gewaltiges geleistet hatten, für ihren Wunsch, sie möge jetzt doch sterben, nicht tadeln sollte. Gleichwohl standen hier das Tötungsverbot und das Lebensrecht der Greisin, die im übrigen nach (nicht aktenkundiger) Erinnerung des Verfassers nicht mit einer PEG-Sonde versorgt war, jeglichem Behandlungsabbruch entgegen.

9 AG Nidda 6 XVII 160/95, insoweit eingehend dargestellt in Seichter aaO (Fn. 7) S. 142.

10 Vgl. Fallbeschreibung Franziska Salver, nachfolgend Fall 8.

11 Auf die heftig umstrittene Frage, ob und auf welcher Rechtsgrundlage eine solche Entscheidung überhaupt zulässig ist, soll hier nicht eingegangen werden.

12 AG Nidda 6 XVII 339/03. 


\section{b) Fallbeispiel für Behandlungsunterlassung bei einem selbst nicht erklärungsfähi- gen Patienten im Sinne passiver Sterbehilfe}

Fall 4:

Der weit über 90-jährige Patient war offensichtlich nicht mehr allzu weit vom Tode entfernt. Wegen mäßiger Atembeschwerden sollte eine Bronchoskopie vorgenommen werden, die aber als mögliche Komplikation dazu hätte führen können, dass der Patient für den Rest seines Lebens intubiert hätte bleiben müssen.

Dem um Rat fragenden Betreuer wurde nachdrücklich geraten, dem Eingriff um dieser Komplikationsgefahr willen nicht zuzustimmen - auch um den Preis des hierdurch möglicherweise sich beschleunigenden Todeseintritts. Denn die Dauerintubation hätte zu einer unvertretbaren Belastung des Lebensrestes des Betroffenen geführt. Dies war übrigens auch durchaus im Sinne der behandelnden Ärzte, die lediglich aus haftungsrechtlichen Gründen eine betreuungsrechtliche Entscheidung gewünscht hatten (»Rechtfertigungsmedizin «).

Wenige Tage später schlug das Krankenhaus zur Linderung der Atemnot eine Lungenpunktion vor, die nicht die Gefahr andauernder Intubierung mit sich brachte. Hier war die Einwilligung problemlos. ${ }^{13}$

\section{c) Fallbeispiele bei vom Patienten ausgehendem Abbruchwunsch}

\section{Fall 5:}

Die 82-jährige nicht mehr einwilligungsfähige Patientin hatte ein Gangrän am Bein, das nach ärztlichem Urteil alsbald zu einer tödlichen Blutvergiftung führen würde, wenn das Bein nicht amputiert würde. Der Allgemeinzustand der Patientin wurde ärztlicherseits als moribund ${ }^{14}$ bezeichnet. Nach Rückfrage mit dem zuständigen katholischen Pfarrer, der angab, die Patientin sei auf das Sterben vorbereitet, lehnte der Richter die Genehmigung der Amputation gegen den Widerstand des einzig erreichbaren Angehörigen ab. Rechtsgrundlage war hier, da ein Betreuer noch nicht bestellt war, § 1846 BGB.

Aus den Gründen: Die Betroffene ist in einem schlechten Allgemeinzustand, aufgrund dessen zu erwarten ist, dass sie nicht mehr lange zu leben hat. Aufgrund dieses Allgemeinzustands ist das Operationsrisiko erhöht, was den lebensverlängernden Erfolg dieses Eingriffs in Frage stellt. ... Die Betroffene hatte sich in der Vorwoche strikt gegen eine Amputation des Beines gewandt. Das Gericht wertet diese Willensäußerung, auch nach Anhörung des einzig erreichbaren Angehörigen der Betroffenen, nicht als mangelnde Einsichtsfähigkeit, sondern als den Wunsch, angesichts des nahenden Todes nicht noch einer derartigen Operation unterzogen zu werden. Diesem aufgrund des vorher Gesagten durchaus nachvollziehbaren Wunsch der Betroffenen muss entsprochen werden. Dass sich die Betroffene zwischenzeitlich nicht mehr äußern kann, führt zu keiner anderen Beurteilung. Schließlich muss berücksichtigt werden, dass eine gegen ihren Willen durchgeführte Amputation die

13 Fallbeispiel aus Seichter aaO (Fn. 7) S. 137.

14 D. h., der Sterbeprozess hat bereits eingesetzt, oder sein Einsetzen steht unmittelbar bevor. 
Betroffene psychisch schwer belasten und damit einerseits den Tod noch schneller herbeiführen, vor allem aber die noch verbleibende Lebensspanne mit Traurigkeit und Depression überschatten würde. ${ }^{15}$

In diesem Fall hatte aus Sicht des Verfassers der Sterbeprozess bereits eingesetzt, so dass insoweit dem Abbruch lebenserhaltender und -verlängernder Maßnahmen nichts entgegenstand. In der Verweigerung der Amputation in der Vorwoche lag eine mündliche (!) sowie zeit- und situationsnahe Patientenverfügung, der damit entsprochen werden konnte.

Fall 6:

Der Richter wurde an einem Freitag in ein Altenheim gerufen mit der Frage, ob die Patientin, die auszutrocknen drohte, zur Sicherstellung der Flüssigkeitsversorgung mit einer PEG-Sonde versorgt werden sollte. Die Patientin habe erklärt, sterben zu wollen. Sie wiederholte diesen Wunsch mit letzter Kraft dem Richter gegenüber. Danach wandte sie sich ab und sprach nichts mehr. Nach Beratung mit Arzt, Pflegepersonal und den beiden über 50-jährigen Töchtern der Betroffenen bestimmte der Richter gemäß $§ 1846$ BGB, dass bis zum Montag eine Versorgung mit einer PEG-Sonde unterbleiben solle. Allerdings seien die Lippen feucht zu halten, auf Wunsch solle selbstverständlich auch zu trinken gegeben werden (Basisbetreuung im Sinne der Grundsätze der Bundesärztekammer zur ärztlichen Sterbebegleitung ${ }^{16}$ ). Um es nicht zu einem qualvollen Verdurstenstod kommen zu lassen, solle gegebenenfalls am Montag über das Legen einer Infusion oder einer PEG-Sonde entschieden werden. Die Betroffene konnte ihrem Wunsch entsprechend noch am Wochenende sterben. ${ }^{17}$

Auch dies wieder ein Fall einer mündlichen Patientenverfügung, die, wenn auch mit letzter Kraft, dem Richter gegenüber wiederholt worden war.

Der Wunsch eines »einfach friedlich Einschlafens « erfüllt sich im übrigen auch dann nicht ohne weiteres, wenn dem Wunsch auf Absetzen der lebenserhaltenden Maßnahmen entsprochen wird. In einem solchen Fall wurde dem Verfasser von einem mehrtägigen schweren Todeskampf berichtet.

Fall 7:

Der über 70-jährige Patient hatte einen Schlaganfall erlitten, eine Verständigung war phasenweise noch möglich. Er wünschte zu sterben. Seine Frau, die ihn ersichtlich liebte, bat, ihm diesen Wunsch zu erfüllen.

Hier wurde ein gemeinsames Gespräch mit der inzwischen als Betreuerin eingesetzten Ehefrau und dem Chefarzt geführt. Arzt und Richter waren sich einig, dass hier aktive Sterbehilfe gewünscht wurde, die nicht gewährt werden kann. Der letztlich auch hier gefundene Konsens ging dahin, dass im Bedarfsfall zur Linderung etwaigen schweren Leidens auch Medikation gewährt werden können sollte, die als Nebenfolge möglicherweise das Leben verkürzen konnte. ${ }^{18}$ Ein solches Vorgehen, das keine finale Tötung darstellt, aber um der Leidenslinderung erforderlichenfalls Lebensverkürzung

15 Fallbeispiel aus Seichter aaO (Fn. 7) S. 136.

$16 \mathrm{aaO}$ (Fn. 4).

17 Fallbeispiel aus Seichter aaO (Fn. 7) S. 137.

18 Fallbeispiel aus Seichter aaO (Fn. 7) S. 136. 
in Kauf nimmt, wird als sogenannte »indirekte Sterbehilfe«, also der Hilfe beim Sterben unter Ausschluss seiner gezielten Herbeiführung, allgemein für zulässig gehalten. ${ }^{19}$

\section{d) Fallbeispiele von Behandlung entgegen dem Willen des Patienten}

\section{Fall 8:}

Franziska Salver (Name geändert), ehemalige Oberärztin, befindet sich nach einem Schlaganfall mit anschließender Hirnblutung in einem Zustand schwerer geistiger Behinderung, der nach neurologischem Urteil mit hoher Wahrscheinlichkeit dauerhaft ist. Sie ist nicht mehr kommunikationsfähig, signalisiert aber Abwehr gegen medizinische und pflegerische Maßnahmen. Manchmal weint sie. Sie ist mit einer PEG-Sonde versorgt. Sie hat eine Patientenverfügung verfasst, in der sie u. a. bestimmt hat, dass lebenserhaltende Maßnahmen unterbleiben sollen, wenn »... die Wahrscheinlichkeit dafür spricht, dass ich eine schwere Dauerschädigung meines Gehirns davontrage, die mir ein menschenwürdiges Dasein nicht mehr erlaubt.«»In guten Tagen« hat sie ihre beste Freundin bevollmächtigt, ihren in der Patientenverfügung enthaltenen Willen für sie durchzusetzen. Zunächst erscheint fraglich, ob Franziska Salver ihren jetzigen Zustand als menschenwürdiges Dasein im Sinne ihrer Patientenverfügung beurteilt hätte. Daraufhin durchgeführte Befragungen in dem Krankenhaus, in dem sie gearbeitet hat, ergeben, dass sie nach Visiten immer wieder gesagt hat, so wie dieser oder jener Patient wolle sie nicht enden. Das habe sich nicht nur auf Koma-Patienten bezogen. Aus alledem wird die Überzeugung abgeleitet, dass Franziska Salver genau den Zustand, in dem sie sich jetzt befindet, für nicht menschenwürdig hält, obwohl sie sich weder im eigentlichen Sterbeprozess befindet, noch ohne Bewusstsein ist. Gleichwohl scheut sich die als Bevollmächtigte eingesetzte Freundin, den Behandlungsabbruch durchzusetzen: Sie hofft, dass es vielleicht doch noch besser wird. Auf Rat der Ethikkommission wendet sich Frau Salvers Freundin nun an das Vormundschaftsgericht. Der Betreuungsrichter gibt erst nach langem Zögern und Einholung eines weiteren ärztlichen Gutachtens die Genehmigung, die Kalorienzufuhr allmählich zu reduzieren und schließlich nur noch Flüssigkeit zu geben. Sechs Wochen später stirbt die Patientin. Seit dem Schlaganfall waren vier Jahre vergangen. ${ }^{20}$

In dem Fall Franziska Salver kann entweder vom Vorliegen einer klaren Patientenverfügung ausgegangen werden, wenn man die Zweifel, was unter »menschenwürdigem Dasein « zu verstehen sein soll durch die Befragungen in ihrem Krankenhaus als ausgeräumt sieht. Zumindest aber haben diese Befragungen so starke Anhaltspunke für einen eindeutigen mutmaßlichen Willen der Patientin erbracht, dass in diesem Fall der ansonsten durchaus zutreffende Vorwurf, bei dem mutmaßlichen Willen handle es sich tatsächlich um keinen Willen, sondern um eine reine Mutmaßung ${ }^{21}$ nicht gerechtfertigt ist. Als Frage bleibt, ob diesem Behandlungsabbruchwunsch entsprochen werden durfte. Oder, allgemeiner gesagt: Beinhaltet das Selbstbestimmungsrecht ein

19 Tröndle - Fischer, Strafgesetzbuch und Nebengesetze, 51. Auflage, Randnummer 18 vor $\S 211$ StGB; BGHSt 42, 301, 305.

20 Fallbeispiel im Internet abgelegt unter www.chrismon.de/ctexte/2003/6/6-2.html. 
Recht der Verfügung über das eigene Leben? Ist hinsichtlich seines eigenen Lebens jeder selbst - Richter über Leben und Tod? Oder kennt die Rechtsordnung bei der Verfügung über Leben auch dann Grenzen des Selbstbestimmungsrechts, wenn es um das eigene Leben geht? Bevor dieser Frage nachgegangen wird, noch ein Fallbeispiel, das zeigt, dass die Antwort vielleicht so einfach nicht ist, wie es auf den ersten Blick scheint.

\section{Fall 9:}

Die 73-jährige Patientin, die u. a. an Bluthochdruckkrankheit, koronarer Herzkrankheit und Niereninsuffizienz mit Schrumpfniere litt, fiel in einen Zustand, in dem sie sediert, intubiert und beatmet werden musste. Die Tochter legte einen Zettel vor, auf dem sich handschriftlich u. a. die Worte »keine lebensverlängernden Maßnahmen, keine Apparate, keine Beatmung, keine Transplantation, keine Abhängigkeit von Maschinen « befanden. Der Zettel war nicht unterzeichnet, es stand aber offenbar außer Frage, dass er von der Patientin herrührte. Den Ärzten fiel ein angespanntes MutterTochter-Verhältnis mit Anhänglichkeit einerseits und Ablösungsbedürfnis andererseits sowie wechselseitiger Abhängigkeit auf. Die Behandlung wurde fortgesetzt, $u$. a. mit täglicher Dialyse. Nach 5 Tagen erwachte die Patientin und extubierte sich selbst. Sie entwickelte eine Lungenentzündung und bat wiederholt, die Hände erhebend: »Lasst mich doch sterben«. Die Bitte wurde von den Ärzten als offenbar ernstlich eingestuft. (Also kein Fall aufgehobener Erklärungsmöglichkeit!) Die Frage, ob der eigentliche Patientenwunsch auf ein Ende des Leidens gerichtet sei, aber nicht auf das Ende des Lebens blieb undiskutiert, weil mit der Patientin wegen Atembeschwerden die Kommunikation hochgradig erschwert war. Unter intensiver Behandlung mit Einlage einer Koronarendoprothese in die linke Nierenarterie mit »fragloser « Einwilligung der (beachte: grundsätzlich einwilligungsfähigen!) Patientin konnte die Patientin auf die Normalstation verlegt werden. Bei einem schweren Rückfall nach 10 Tagen beharrte die Patientin einerseits auf Therapieverzicht, war aber einer Rückverlegung auf die Intensivstation gegenüber gleichwohl nicht abgeneigt. Eine entsprechende Ambitendenz war bei der Tochter festzustellen, möglicherweise, weil sie bei einer weiteren Verschlechterung von einer Intensivbehandlung Leidensminderung erwartete. Ohne definitive Entscheidung der an sich bewusstseinsklaren Patientin verlegte die behandelnde Ärztin diese von sich aus wieder auf die Intensivstation. Nach ihrer Einschätzung würde die Patientin nicht sterben können, ohne den Todeskampf mit vollem Bewusstsein mitzuerleben. In dieser Phase konnte am Krankenbett die Erkenntnis vermittelt werden, dass es einen einfachen, geschenkten Tod nicht gibt. Letztlich genas die Patientin und lebt nun 2 Jahre nach diesen Ereignissen in einem Altenheim. Rückblickend erklärt sie bei nur noch geringer Ambivalenz hinsichtlich der an ihr vorgenommenen Weiterbehandlung, wenn sie nicht überlebt hätte, wäre das »doch auch gut« gewesen. Andererseits hätte ihr schon das Überleben für einige

21 Sondervotum Beckmann, B1. 59 im Zwischenbericht»Patientenverfügungen« der EnqueteKommission Ethik und Recht der modernen Medizin vom 13.09.2004, Bundestags-Drucksache 15/3700, pdf-Download im Internet unter www.bundestag.de/medizin. 
Wochen viel bedeutet, weil sie in dieser Zeit viel mit der Tochter habe besprechen können, war bisher unausgesprochen geblieben sei. ${ }^{22}$

Die rechtliche Bewertung des Vorgehens der Ärzte in diesem dramatischen Fall ist heikel. Vom guten Ergebnis her möchte man es billigen. Aber wo ist denn hier die Berücksichtigung des Willens, des Selbstbestimmungsrechts der ja offenbar weitgehend einwilligungsfähigen Patientin? Auf diese weit über den geschilderten Einzelfall hinaus relevante Frage wird nachfolgend noch einzugehen sein.

\section{Die Entscheidung des Bundesgerichtshofs vom 17.03.2003}

\section{a) Darstellung der Entscheidung}

In seinem lange erwarteten Grundsatzbeschluss vom 17.03.2003 ${ }^{23}$ hat der Bundesgerichtshof über die Frage entschieden, ob und wann eine Patientenverfügung, die bei irreversibel tödlichem Krankheitsverlauf lebenserhaltende oder -verlängernde Maßnahmen ablehnt, für den Arzt bindend ist. Im Ergebnis unterscheidet er dabei folgende Fallgruppen:

(1) Hat der (inzwischen einwilligungsunfähige) Patient zu einem Zeitpunkt, als er noch einwilligungsfähig war, explizit erklärt, lebenserhaltende und -verlängernde Maßnahmen abzulehnen, muss dem entsprochen werden.

(2) Ist eine ausdrückliche Willenserklärung des Patienten nicht bekannt, beurteilt sich die Zulässigkeit der Behandlung, falls unaufschiebbar, nach dem mutmaßlichen Willen des Patienten, bis für diesen ein Betreuer bestellt ist.

(3) Ist ein Betreuer bestellt und erreichbar, stellt der mutmaßliche Wille des Patienten keine zureichende Behandlungsgrundlage mehr dar, weil durch die Einsetzung des Betreuers die unmittelbare rechtliche Handlungsfähigkeit des Patienten wiederhergestellt ist. Hier hat der Betreuer als gesetzlicher Vertreter des Patienten die erforderlichen Erklärungen abzugeben, wobei er gemäß § 1901 BGB den Willen des Patienten in eigener Verantwortung zu berücksichtigen und erforderlichenfalls durchzusetzen hat.

(4) Liegt nach ärztlichem Urteil keine medizinische Indikation für lebenserhaltende und -verlängernde Maßnahmen vor, darf der Arzt von sich aus solche Maßnahmen unterlassen. Hierzu bedarf es weder der Einwilligung des Betreuers, noch einer Genehmigung durch das Vormundschaftsgericht. Insoweit liegen Dinge nicht anders als bei einwilligungsfähigen Patienten, dessen Behandlungswunsch der Arzt ablehnen darf, wenn es an einer entsprechenden Indikation fehlt.

(5) Bejahen die Ärzte die Indikation für eine Weiterbehandlung, ist nach allgemeinen Grundsätzen die Einwilligung des Betreuers erforderlich.

22 Dieser Fall wird (auch medizinisch) ausführlich berichtet und erörtert von Spittler/Fritscher-Ravens, Deutsche Medizinische Wochenschrift 2001, 925. Vgl. hierzu auch Czerner, Legitimierung von Behandlungsabbrüchen durch § 1904 BGB?, KritV 2/2004, 182 ff.

23 aaO (Fn. 3). 
(6) In den Fällen, in denen der Betreuer eine Weiterbehandlung ablehnt, obwohl sie ärztlicherseits für indiziert angesehen wird (»Konfliktfall«), bedarf diese ablehnende Entscheidung des Betreuers der vormundschaftsgerichtlichen Genehmigung. Anders ausgedrückt: das Vormundschaftsgericht ist überhaupt nur in den Fällen zu beteiligen, in denen die Ärzte in ihrer eigenen Verantwortung eine Weiterbehandlung für richtig halten und der Betreuer gleichwohl den Abbruch der Behandlung wünscht.

Daraus wird deutlich, dass der BGH dem Arzt einen erheblichen Entscheidungsspielraum lässt. ${ }^{24}$

\section{b) Tödlicher Verlauf auch vor »unmittelbarer Todesnähe«?}

Aufgrund einer in Abschnitt III. 2. c) aa) der soeben dargestellten Entscheidung erfolgten Bezugnahme auf den $»$ Kemptener Fall ${ }^{25}$ war der Eindruck entstanden, dass ein Behandlungsabbruch nur »in unmittelbarer Todesnähe« in Betracht kommt.

Diese Begrenzung ist von Bühler/Stolz $z^{26}$ aus ärztlicher Sicht heftig kritisiert worden. Sie schlagen vor, von einem irreversibel tödlich verlaufenden Grundleiden (und damit einem »tödlichen Verlauf« im Sinne des BGH-Beschlusses) auszugehen, wenn folgende Merkmale vorliegen:

(1) bei irreversibler Bewusstlosigkeit und Kommunikationsunfähigkeit;

(2) bei Schwerstpflegebedürftigkeit;

(3) bei der Notwendigkeit medizinisch indizierter künstlicher Unterstützung von Nahrungsaufnahme, »Entgiftung « und Ausscheidung oder Beatmung;

(4) rasches Ableben bei Beendigung dieser künstlichen Unterstützungsmaßnahmen ist mit Sicherheit zu erwarten.

Dagegen solle kein Behandlungsabbruch in Betracht kommen,

- wenn eine völlige Wiederherstellung (restitutio ad integrum) möglich sei,

- bei chronischen Leiden (wie Diabetes mellitus, Asthma, Rheuma), die auch bei Behandlung lege artis zwar zu Folgeerscheinungen mit tödlichem Ausgang führen könnten, deren Komplikationen aber im Regelfall durch geeignete Maßnahmen aufgehalten und vermieden werden könnten

- bei Leiden, bei denen die Patienten sich noch selbst ernähren, spontan atmen, sich bewegen könnten und bei denen die Patienten noch selbst entscheiden könnten, ob sie Nahrung und Flüssigkeit aufnähmen.

Bei solchen Erkrankungen werde nämlich die emotionale, faktische und wirtschaftliche Belastung durch die Krankheit in der Regel nicht als hoffnungslos, würdelos und unerträglich empfunden. In diesen Fällen bedeute eine Lebensverlängerung durch die möglichen medizinischen Maßnahmen keine (bloße) Leidensverlängerung.

In einem Interview mit der $\mathrm{FAZ}^{27}$ hat die Vorsitzende des Senats, der den in Rede stehenden Beschluss erlassen hat, erklärt, der Senat habe an der genannten Einschrän-

24 Borasio/Putz/Eisenmenger aaO (Fn. 5); ebenso für die Sterbephase schon Erman - A. Roth, 10. Auflage, § 1904 BGB Rdnr. 22.

25 aaO (Fn. 8).

26 FamRZ 2003, 1622; 
kung nicht festhalten, sondern den Behandlungsabbruch bei irreversibel tödlichem Verlauf auch vor Eintritt der eigentlichen Sterbephase erfassen wollen. Wo keine Chance bestehe, »dass der Patient wieder ... als bewusster Mensch am Leben der anderen teilnehmen kann, ... befindet sich u. E. ein solcher Wachkomapatient in einem irreversiblen tödlichen Verlauf.«Zwar handelt es sich bei dieser Äußerung im Rechtssinne um eine Privatmeinung, noch unterhalb eines obiter dictum. Inzwischen hat aber das OLG Karlsruhe in einem Beschluss vom 26.03.2004 ${ }^{28}$ ausdrücklich ausgesprochen, dass ein Behandlungsabbruch nach den vom BGH gesetzten Vorgaben auch in Betracht kommen kann, wenn das Leiden des Patienten einen irreversiblen tödlichen Verlauf genommen hat, der Tod aber nicht unmittelbar bevorsteht.

\section{c) Unzulässige Beschneidung des Selbstbestimmungsrechts des Patienten?}

Die Entscheidung des BGH ist immer wieder im Hinblick darauf kritisiert worden, sie enge das Selbstbestimmungsrecht des sterben-wollenden Patienten zu stark ein.

Teilweise beruht diese Kritik allerdings auf Unkenntnis dieses Beschlusses oder dessen falscher Interpretation. Wenn etwa die Deutsche Hospiz Stiftung beklagt, schriftliche Patientenverfügungen reichten in der Regel zur Beendigung lebensverlängernder Maßnahmen nicht mehr aus ${ }^{29}$, trifft dies, wie die vorstehende Darstellung zeigt, in dieser Allgemeinheit nicht zu.

Anders verhält es sich mit der Kritik des CSU-Bundestagsabgeordneten Dr. Martin Mayer (Siegertsbrunn) ${ }^{30}$ an dem Zwischenbericht Patientenverfügung der EnqueteKommission Ethik und Recht in der modernen Medizin des Deutschen Bundestages ${ }^{31}$, das Grundrecht auf körperliche Unversehrtheit werde in den Empfehlungen der Enquete-Kommission dadurch zu sehr eingeschränkt, dass Patientenverfügungen mit dem Ziel der Beendigung lebenserhaltender und -verlängernder Maßnahmen nur in den Fällen wirksam sein sollten, »in denen das Grundleiden irreversibel sei und trotz medizinischer Behandlung nach ärztlicher Erkenntnis zum Tod führen werde«. Damit würden Demenzkranke, Wachkomapatienten und viele Andere von der Möglichkeit, durch eine Patientenverfügung die Beendigung ihrer Behandlung abzulehnen, ausgeschlossen. Das Recht auf Leben schließe aber das Recht auf ein Sterben in Würde und das Recht auf einen natürlichen Tod ein. Patientenverfügungen müssten definitiv bindende Kraft erhalten, wobei als Voraussetzungen hierfür eine gewisse Aktualität und eine vorausgegangene qualifizierte ärztliche Beratung gefordert werden könnten.

In der Tat: Was ist denn mit Patientenverfügungen, die außerhalb der vom BGH gesetzten Voraussetzungen einen Abbruch ärztlicher Maßnahmen fordern? Sind sie unwirksam? Auf welcher Grundlage wird hier das Selbstbestimmungsrecht des Verfü-

27 Hahne im Rechtsinterview mit Rudolf Gerhardt in FAZ v. 18.07.2003, zitiert in Coeppicus, RPfleger 2004, S. 262.

28 BtPrax 2004, 202.

29 Deutsches Ärzteblatt Jg. 100 Heft 31 - 32, vom 4. August 2003, A 2064.

30 Veröffentlicht im Internet unter www.m4m.de/patientenverfuegung/04-09PM-Patientenverfueg-Zwiber.htm.

$31 \mathrm{aaO}$ (Fn. 21). 
genden beschränkt? Liegt in der Beschränkung der Geltung von Patientenverfügungen auf »tödliche Verläufe« (selbst bei Ausdehnung des Begriffs vor die eigentliche Sterbephase) eine grundrechtswidrige Einschränkung des Selbstbestimmungsrechts? ${ }^{32}$

\section{d) Unmittelbare Geltung des in der Patientenverfügung erklärten Willens?}

Coeppicus weist in seinem Aufsatz »Abbruch lebenserhaltender Maßnahmen ${ }^{33}$ auf Folgendes hin:

Auch nach Verlust der Einwilligungsfähigkeit und der Bestellung eines Betreuers bleibt der in einwilligungsfähigem Zustand erklärte Willen des Patienten vollwirksam bestehen. (Auf den Sonderfall, dass Anhaltspunkte für Unrichtigkeit dieser Erklärung von Anfang an vorliegen oder aber dafür, dass der Patient von dem geäußerten Willen abrücken will, soll in diesem Zusammenhang nicht eingegangen werden: Bei Wachkomapatienten wird das nur ausnahmsweise der Fall sein.) Gemäß § 130 Absatz 2 BGB ist es nämlich auf die Wirksamkeit einer Willenserklärung ohne Einfluss, wenn der Erklärende nach deren Abgabe geschäftsunfähig wird. Auf den ausdrücklich erklärten Willen des inzwischen einwilligungsunfähigen Patienten kann der Arzt daher auch nach Bestellung eines Betreuers unmittelbar durchgreifen, es sei denn, der Betreuer hätte diesen Willen ausdrücklich widerrufen. ${ }^{34}$ Dies ergibt sich auch aus dem Beschluss des BGH, in dem die Einsetzung eines Betreuers lediglich den Durchgriff auf den mutmaßlichen Willen des Patienten ausschließt. ${ }^{35}$ Damit ist, jedenfalls beim Vorliegen einer unmissverständlich abgefassten Patientenverfügung, weder eine Einwilligung des Betreuers, noch eine Genehmigung durch das Vormundschaftsgericht erforderlich. Denn eigene Erklärungen eines voll Geschäftsfähigen bedürfen in keinem Fall einer Genehmigung durch das Vormundschaftsgericht. Wenn der Patient selbst entscheiden kann, dann gilt sein Wille ohne Rücksicht auf die in dem BGHBeschluss gesetzten Grenzen. ${ }^{36}$

Es ist anerkanntes Recht, dass das Selbstbestimmungsrecht auch die Befugnis umfasst, ärztliche Behandlungen abzulehnen. Das gilt sogar, wenn die Ablehnung objektiv unsinnig ist, womöglich mit tödlichem Ausgang, während eine Behandlung zur vollständigen Wiederherstellung führen würde. ${ }^{37}$

Schließlich zeigt Coeppicus auch noch dogmatische Ungereimtheiten auf, die eintreten, wenn weder die Einwilligung des Patienten selbst, noch die des Betreuers in die weitere Behandlung vorliegt. Der BGH-Beschluss stellt insoweit eher beiläufig fest, dass die Ablehnung des vom Betreuer angestrebten Behandlungsabbruchs durch das Vormundschaftsgericht zugleich die Einwilligung in die weitere Behandlung enthält. Allerdings gibt es hierfür keine rechte gesetzliche Grundlage. Ein Fall des $§ 1846$ BGB liegt nicht vor, denn ein Betreuer ist ja vorhanden und offenbar gerade nicht ver-

32 So Coeppicus, RPfleger 2004, 262.

33 aaO (Fn. 32).

34 Auch dazu ist er befugt!

35 Abschnitt III. 2. a) des BGH-Beschlusses.

36 Kutzer, ZRP 2003, 213.

37 Coeppicus aaO (Fn. 32) mwN. 
hindert. So mag auch dieser Ausspruch Antwort auf ein unabweisbares Bedürfnis des Betreuungsrechts sein, auf das der Beschluss sich ja, wenn auch in anderem Zusammenhang, stützt. Ebenso bleibt vor diesem Hintergrund offen, weshalb und auf welcher Einwilligungsgrundlage auch beim Vorliegen einer »klipp und klaren« Patientenverfügung bis zum Abschluss des vormundschaftsgerichtlichen Verfahrens weiterbehandelt werden muss. $^{38}$

\section{e) Grenzen des auf Lebensbeendigung hin gerichteten Selbstbestimmungsrechts}

Die bereits angesprochene auch von dem BGH-Beschluss aufgenommene Rechtsprechung, wonach jeder Kranke das Recht hat, sich nicht behandeln zu lassen, führt in der Tat letztlich zur Disponibilität auch des eigenen Lebens. Dieses obschon ethisch fragwürdige Ergebnis wird bei dem erklärungsfähigen Patienten letztlich nicht in Frage gestellt: Der Arzt kann versuchen, ihn zu überreden, sich behandeln zu lassen, vielleicht versucht er, ihn zu täuschen, zu überlisten. Letztlich zwingen kann er den Patienten nicht.

Eingeschränkt wird das »Recht auf Krankheit« allerdings zum einen, wenn die Fähigkeit, einen eigenen Willen zu bilden so massiv gestört ist, dass von einer eigentlichen eigenen Willensentscheidung nicht mehr gesprochen werden kann.

Fall 10:

Der Betroffene hat einen Embolus (Blutgerinnsel) im Arm, der mit fatalem Ausgang in die Hirnschlagader geschleudert zu werden droht. Der Embolus könnte nach einem kleinen Einschnitt in den Arm entfernt werden. Hierzu verweigert der Betroffene die Einwilligung. Dem herbeigerufenen Richter erklärt er, er sei an Hodenkrebs erkrankt gewesen und habe in diesem Zusammenhang so üble Operationen über sich ergehen lassen müssen, dass er sich geschworen habe, keinerlei Operation mehr an sich zuzulassen. Der Eingriff wurde analog § 1906 IV BGB betreuungsrichterlich genehmigt. Zur Begründung wurde ausgeführt, der von den Ärzten vorgeschlagene Eingriff sei minimal, sein Unterbleiben könne aber zu unabweisbaren sehr viel schwereren Eingriffen führen und damit genau zu dem, was der Betroffene eigentlich vermeiden wolle. Nur durch den Minimaleingriff, der ja kaum als Operation anzusehen sei, könne seinem Wunsch, eigentlichen Operationen nicht mehr ausgesetzt zu werden, entsprochen werden. ${ }^{39}$

Die zweite Einschränkung findet sich in Fällen von Selbsttötung. Die lebensrettende Behandlung eines Suizidenten ist ja zwangsläufig die Behandlung eines Patienten ohne, ja gegen dessen Willen. Rechtsgrundlage der Behandlung sind hier aber die Gesetze der einzelnen Bundesländer über die Behandlung psychisch Kranker, so dass es dogmatisch in der Tat keiner Einwilligung bedarf. In diesem Bereich ist das Selbstbestimmungsrecht erheblich schwächer, als im Zivil- und Arztrecht: »Das Grundgesetz gewährt über das eigene Leben kein Verfügungsrecht, so dass es keinen verfassungsrechtlich verbürgten Anspruch auf 'aktive Sterbehilfe' durch Dritte geben

38 So aber Kutzer, ZRP 2003, 213.

39 Fallbeispiel aus Seichter aaO (Fn. 7) S. 127. 
kann. Das menschliche Leben ist vielmehr vom Staat zu schützen. Es gehört daher zu den Aufgaben der Polizei, die Tötung eines Menschen zu verhindern, unabhängig davon, ob sie Folge einer Straftat oder lediglich eines Unglücksfalles wäre oder ob sie sich als straflose Selbsttötung darstellte. ${ }^{40}$ Auch im Strafrecht findet sich in einer im Jahr 2001 ergangenen Entscheidung des BGH der Satz: »Die Rechtsordnung wertet eine Selbsttötung deshalb - von äußersten Ausnahmefällen abgesehen - als rechtswidrig (und) stellt die Selbsttötung und die Teilnahme hieran lediglich straflos. « ${ }^{41}$

Das Zivilrecht indes ist bestimmt von dem »Recht auf Krankheit« Es bleibt die Frage, ob dieses Recht, dass dem Erklärungsfähigen nach gefestigter Rechtsauffassung zusteht, nach Wegfall der Erklärungsfähigkeit nur noch eingeschränkt gilt.

\section{Stellungnahme zu dem BGH-Beschluss}

Ungeachtet der dogmatischen Ungereimtheiten, wie sie etwa Coeppicus ausgezeigt hat $^{42}$, geht der BGH in seiner Entscheidung ersichtlich davon aus, dass mit dem Erlöschen der Einwilligungsfähigkeit auch eigene Erklärungen des Patienten jedenfalls im Konfliktfall zwischen Betreuer und Arzt der Überprüfung durch den Betreuungsrichter zugänglich sind. Dies ist vom Ergebnis her auch zu begrüßen. Denn die Situation zwischen einem Erklärungsfähigen, der jetzt, aktuell, keine Behandlung wünscht und einem nicht mehr Erklärungsfähigen, der erklärt hat, in einer bestimmten, künftig eintretenden Situation, keine Behandlung zu wünschen, ist unterschiedlich.

a) Der Erklärungsfähige äußert sich in einer Situation, die er kennt. Der Arzt kann seine Situation und seine Entscheidung dazu konkret und substantiiert erörtern. Mögliche Probleme können angesprochen und behoben oder doch gemindert, Ängste ausgesprochen und Wege zu ihrer Bewältigung aufgezeigt werden. Aufgrund dieser Erörterung kann der Patient seine Entscheidung reflektieren und abändern. Todessehnsucht kann neuer Hoffnung weichen, sei sie auch begrenzt.

Nach den weithin bekannten Forschungen von Elisabeth Kübler-Ross gibt es fünf Phasen des Sterbens: die Phase des Nichtwahrhabenwollens, die Phase des Aufbegehrens, die Phase der Depression, die Phase des Verhandelns um noch etwas Zeit und die Phase des sich Fügens in den bevorstehenden Tod. Nicht jeder erlebt alle Phasen, nicht bei jedem kommen sie in dieser Reihenfolge. Aber diese Forschungsergebnisse kennzeichnen das Sterben als einen Prozess, der gelebt und nicht einfach abgebrochen werden sollte. Abgebrochen oft gar nicht wegen des Leidens des Patienten selbst, sondern seiner Angehörigen und vielleicht auch des Pflegepersonals.

Ein Mediziner hat in einem Beitrag zu dem BGH-Beschluss geschrieben: »Ein sehr großer Teil der Menschen ist stimmungslabil, der augenblicklich geäußerte Wunsch zum Sterben entspricht nicht deren dauerhafter Einstellung zum Leben. ... Der Wunsch zu sterben soll als Hilferuf provozieren ... Die demonstrative Aufforderung zur Tötung ist dementsprechend selbst bei Schwerstkranken häufig nicht ernst

40 VG Karlsruhe NJW 1988, 1536.

41 BGH NJW 2001, 1802.

$42 \mathrm{aaO}$ (Fn. 32). 
gemeint. Der Hilferuf erheischt Hilfe, Euthanasie schafft Abhilfe. Aber auch bei Schwerstkranken kann der Wunsch zu sterben von Angehörigen induziert, ja oktroyiert werden. $\ll^{43}$

Alte Menschen haben vielfach Angst, ihren Angehörigen zur Last zu fallen. Vor diesem Hintergrund ein mahnendes Wort von Altbundespräsident Rau: $»$ Wo das Weiterleben nur eine von zwei legalen Optionen ist, wird jeder rechenschaftspflichtig, der anderen die Last seines Weiterlebens aufbürdet. ${ }^{44}$

Solchen Gedanken muss sich die Justiz, muss sich die Gesellschaft stellen. Man widerstehe der Versuchung, sie mit einem Haufen dogmatischer Richtigkeiten zuzuschütten. Erforderlichenfalls, etwa im Hinblick auf die dargestellten unterschiedlichen Wertungen in Polizei- und Strafrecht einerseits und Zivil- und Arztrecht andererseits, müssen sich auch gefestigte Dogmen in Frage stellen lassen.

Ein pensionierter Amtsrichter ${ }^{45}$ hat einen Beitrag zu dem BGH-Beschluss verfasst und ihm die merkwürdig anmutende Überschrift »Recht und Gefühl« gegeben. Gegen Ende seiner Ausführungen schreibt er: »Wann man einen Menschen sterben lassen sollte, kann - im besten Sinne - nur erspürt werden.« Eben dieses »Erspüren« mag in dem vorstehend dargestellten Fall 9 zu der bei strikter rechtlicher Betrachtungsweise durchaus problematischen und mutigen Entscheidung der Weiterbehandlung bewegt haben. Mit der Patientin, die ja weitestgehend erklärungsfähig blieb, war ein entsprechender Dialog zumindest eingeschränkt möglich, bei Wegfall der Erklärungsfähigkeit ist das nicht der Fall.

b) Bei dem nicht mehr Erklärungsfähigen ist die Möglichkeit, die vormals getroffene Entscheidung zu problematisieren und gegebenenfalls abzuändern, naturgemäß aufgehoben oder jedenfalls auf ein Minimum reduziert. Das rechtfertigt, sie in dem von dem BGH geforderten Umfang der Kontrolle durch den Betreuungsrichter zugänglich zu machen. Wie ausgeführt, ist diese Kontrolle ja auf Fälle des Konflikts zwischen Arzt und Betreuer beschränkt. Ist in diesen Fällen der Betreuungsrichter »Richter über Leben und Tod«? Nein, er durchleuchtet lediglich die beiderseits vorgebrachten Gesichtspunkte und versucht den Konflikt aufzulösen ${ }^{46}$ - nur notfalls entscheidet er. Aber zum Richter über Leben und Tod macht ihn seine Entscheidung ebensowenig wie die einen Arzt, der eine bestimmte Therapie anwendet oder eine riskante Operation vornimmt und den Patienten dadurch nicht retten kann.

In nicht wenigen Fällen liegen übrigens durchaus wahrnehmbare Anhaltspunkte für einen Weiterlebenswillen vor. So wäre in vorstehend dargestelltem Fall 1 angesichts einer durchaus noch feststellbaren Lebensfreude verbunden mit dem Fehlen von Todessehnsucht das Legen der PEG-Sonde auch zu genehmigen gewesen, wenn ein früherer entgegenstehender Wille festzustellen gewesen wäre (was ja hier nicht der Fall war). Fall 2 belegt sogar durchaus lebhafte Lebensfreude, auch im Zustand praktisch kompletter Demenz. Bei einer so verlaufenden Demenz wäre auch einer Patien-

43 Förster ZRP 2003, 378.

44 Zitiert in Kutzer, ZRP 2003, 209.

45 Paehler BtPrax 2003, 141.

46 Ebenso Borasio/Putz/Eisenmenger aaO (Fn. 5). 
tenverfügung des Inhalts, dass bei einer Demenz die Behandlung abgebrochen werden solle, unbeachtlich gewesen: die kreatürliche Lebensfreude der Patientin hätte ohne weiteres als konkreter Anhaltspunkt für ein Fallenlassen des früher erklärten Nichtbehandlungswunsches ausgereicht.

Schließlich noch Fall 8 »ranziska Salver«. Hier lag zwar eine Patientenverfügung vor, aber die als Bevollmächtigte eingesetzte beste Freundin der Patientin zögerte, in den Behandlungsabbruch einzuwilligen: Sie hoffte immer noch auf Besserung. An diesem Fall wird die zwangsläufige Schwäche jeder Patientenverfügung deutlich: Sie kommt nicht ohne Hypothesen aus. Die Frage, ob eine Dauerschädigung bleibt, ist keine juristische, sondern eine medizinische, und oftmals nicht schnell zu beantworten. Die Frage, ob die Krankheitssituation einem menschenwürdigen Leben entspricht, ist eine juristische oder ethische, machte aber hier zunächst weitere Ermittlungen erforderlich. Der zusätzlich Zeit und Geld kostende Einsatz eines Verfahrenspflegers wird in derlei Fällen zwar nicht von allen Betreuungsrichtern eingehalten, von den Obergerichten aber durchaus verlangt ${ }^{47}$.

c) Es kann und soll nicht bestritten werden, dass die Fortschritte der Medizin ein Weiterleben früher (und auch heute noch in anderen Ländern) längstens dem Tode Geweihter ermöglicht, bei denen in der Tat der Sterbeprozess in unethischer Weise hinausgeschoben zu werden droht und auch hinausgeschoben wird. Die Notwendigkeit zu definieren und eben auch zu entscheiden, wann das Therapieziel von Lebenserhaltung und -verlängerung auf Schmerz- und Beschwerdenlinderung umzustellen ist, und wie und durch wen diese Entscheidung zu treffen ist, ist unübersehbar.

In der Medizinethik wird zu Recht immer wieder diskutiert, ob alles, was medizinisch möglich ist, auch erlaubt ist. Bei der Frage der Therapiebegrenzung geht es aber um die Frage, oder der Arzt zu allem, was medizinisch möglich ist, verpflichtet sein soll, vielleicht sogar unter Strafandrohung (»Rechtfertigungsmedizin «). Dies darf nicht der Fall sein, wenn bei bereits weitestgehend eingetretenem Lebensverlust lebenserhaltende Unterstützung ihren Sinn verloren hat. ${ }^{48}$

Neben dem verfassungsrechtlich geschützten Recht auf Leben wird zunehmend die Forderung nach einem Recht auf Sterben laut. Dabei geht es auch nicht allein nur um die Angst vor dem Leiden und dem Todeskampf, sondern um die Angst vor einem mechanisch Am-Leben-Erhalten-Werden, obwohl das, was das Leben ausmacht, bereits erloschen ist, um die Angst vor einem »schon tot noch lebendig sein zu müssen«. Vielfach wird man hier einer Patientenverfügung erhebliche Bedeutung einräumen können und müssen: Bei Fragen etwa, ob man als Wachkomapatient die letztlich nie völlig auszuschließende Chance des Wiederaufwachens ungenutzt lassen möchte, oder wenn man nach ärztlichem Urteil voraussichtlich keine Möglichkeit mehr haben wird, mit der Umwelt in Kontakt zu treten, die Einstellung lebenserhaltender Maßnahmen wünscht, erscheint ein »Nein « ethisch vertretbar und sollte durchaus berücksichtigt werden. Vergleiche hierzu auch die vorstehenden Fälle 5 und 6.

47 Z. B. OLG Karlsruhe, Fn. 28.

48 Ebenso Paehler, BtPrax 2000, 21. 
Die dargestellten Unterschiede zwischen der aktuellen Erklärung eines einwilligungsfähigen Patienten und einer früheren Erklärung eines Patienten, der inzwischen seine Einwilligungsfähigkeit verloren hat, rechtfertigt, ja gebietet, den Erklärungen in einer Patientenverfügung keine absolute Bindungswirkung zu geben, sondern sie in dem Umfang, wie es der BGH für Erklärungen eines Betreuers entschieden hat, vormundschaftsgerichtlicher Überprüfbarkeit zu unterstellen. Der BGH beschränkt diese Überprüfung ja bei festgestelltem irreversiblem tödlichen Verlauf auf den Konfliktfall, in dem Arzt und Betreuer sich nicht einigen können. Frühere Einwilligungen des Patienten selbst in einer Patientenverfügung sollten darüberhinaus überprüfbar gestellt werden, in denen Anhaltspunkte dafür bestehen, dass der vormals erklärte Therapiebeendigungswille nicht mehr aufrechterhalten wird. Bei noch einwilligungsfähigen Patienten ist häufig auch nach Erhalt einer infausten Prognose ein verbissener Überlebenswille, ein Ringen um Monate und Wochen, festzustellen. Dieses Kriterium wird bei nicht mehr einwilligungsfähigen Patienten meist nicht eruierbar sein. Hier bieten sich als Kriterium an das Vorliegen noch einer Vielzahl von Lebensäußerungen (vgl. oben Fall 2) und/oder das Fehlen von Anhaltspunkten für Todessehnsucht, für ein das Leben-Aufgeben-Wollen. Der Verfasser ist mit Paehler ${ }^{49}$ der Auffassung, dass solches in vielen Fällen auch bei einwilligungsunfähigen Patienten, insbesondere für erfahrene Ärzte und Pfleger, durchaus »erspürt« werden kann. Für die Feststellung eines »irreversibel tödlichen Verlaufs « bietet der Aufsatz von Bühler/Stolz ${ }^{50}$ eine ethisch begründete und praktisch umsetzbare Grundlage.

Als dogmatische Anknüpfung für die Überprüfbarkeit des Inhalts von Erklärungen in Patientenverfügungen bietet sich die Aufnahme des Rechtsgedankens aus $\S 1904$ Absatz 2 und $\S 1906$ Absatz 5 BGB an. In diesen beiden Bestimmungen sind Entscheidungen der gerichtlichen Überprüfung unterstellt, die von durch den Patienten selbst eingesetzten Bevollmächtigten getroffen werden und nicht von einem vom Gericht eingesetzten Betreuer. Diese Situation lässt sich zwanglos auf Erklärungen in einer Patientenverfügung eines inzwischen Erklärungsunfähigen übertragen, sei es durch Analogie oder aufgrund eines unabweisbaren Bedürfnisses des Betreuungsrechts. Anknüpfung für diese Unabweisbarkeit in dem dargestellten Umfang ist die Verpflichtung des Staates zum Lebensschutz aus Artikel 2 Absatz 2 GG, die nach Artikel 1 Absatz 3 Grundgesetz auch die Rechtsprechung als unmittelbar geltendes Recht bindet.

Die an sich erforderliche Einwilligung des Patienten selbst in die Weiterführung der Behandlung liegt, soweit das Gericht die Genehmigung des Behandlungsabbruchs ablehnt, notwendigerweise unmittelbar in dieser Gerichtsentscheidung. So ist es auch in dem BGH-Beschluss ausgeführt. Die Befugnis des Gerichts hierzu ergibt sich als notwendiges Annex aus der Befugnis, den Behandlungsabbruch zu verweigern. Damit liegt ein Sonderfall außerhalb $\S 1846$ BGB vor, so dass der Einwand von Coeppicus $^{51}$, der Betreuungsrichter sei nicht befugt, bestimmte Angelegenheiten gegen den Willen des Betreuers »in seinem Sinne« zu regeln, nicht Platz greift.

49 BtPrax 2003, 141.

50 aaO (Fn. 26; vgl. dessen auszugsweise Wiedergabe oben S. 459.).

$51 \mathrm{aaO}$ (Fn. 32). 
Was das vom Richter zu beachtende Verfahren angeht, sollte dies weitgehend in dessen Ermessen gestellt werden. Wie ausgeführt geht es bei der genannten Überprüfung ihrem Wesen nach um eine zusammenfassende Erörterung der unterschiedlichen Aspekte unter- und gegeneinander unter Leitung, vielleicht auch Mediation des Richters $^{52}$. Es reicht aus, dieses Gespräch zu dokumentieren ${ }^{53}$. Inwieweit der Richter in den häufig evidenten Fällen noch zusätzliche externe Begutachtungen veranlassen oder Verfahrenspfleger beteiligen soll, sollte ihm überlassen bleiben. Allenfalls wäre an ein entsprechendes Antragsrecht der Beteiligten zu denken, das den Richter binden könnte. Grenzsituationen wie die Überprüfung, ob einem Wunsch auf Behandlungsabbruch entsprochen werden kann, entziehen sich weitgehend der für das rechtliche Denken üblichen und ja auch erforderlichen Typisierung und Generalisierung. Das ist übrigens im Familienrecht, dem das Betreuungsrecht zugehört, nicht selten. Aus diesem Grund ist ein zwingend vorgegebenes förmliches Verfahren unsachgerecht und sollte weder dem Richter, noch den Beteiligten aufgenötigt werden - unter anderem um den Preis einer wochen- oder gar monatelangen Verfahrensdauer.

\section{Schlussfolgerungen für die Abfassung von Patientenverfügungen}

Der in einer Patientenverfügung enthaltene Wunsch, beim Eintritt bestimmter Umstände sollten lebensverlängernde und -erhaltende Maßnahmen unterbleiben oder abgebrochen werden, ist im BGH-Beschluss umrissenen Umfang grundsätzlich wirksam.

Diese Wirksamkeit ist umso stärker, je näher dem Patienten beim Abfassen der Patientenverfügung die Situation, die er darin beschreibt, ist. Die Verfügung eines 20jährigen, im Falle seiner Altersdemenz wolle er keine Weiterbehandlung, hat evident weniger Kraft, als die Verfügung eines Patienten, der nach Stellung der Diagnose morbus Alzheimer Bestimmungen für das Endstadium seiner Erkrankung trifft. Besondere Kraft wird eine Patientenverfügung haben, die aufgrund eines eingehenden Aufklärungsgesprächs mit einem behandelnden Arzt verfasst und von diesem mit unterzeichnet wird. Damit steht dieser erforderlichenfalls auch für Rückfragen bei Unklarheiten zur Verfügung.

Die Bestimmungen in der Patientenverfügung sollten möglichst konkret und klar sein. Die oft anzutreffende Verfügung, man wünsche keine Behandlung, wenn ein »menschenwürdiges Leben « nicht mehr möglich sei, ist zu unklar und entsprechend auslegungsfähig.

Eine absolute Bindungswirkung kann eine Patientenverfügung nicht beanspruchen. Die Möglichkeit einer Änderung der vormaligen Meinung kann nie ausgeschlossen werden. Zwar reicht allein diese abstrakte Möglichkeit nicht aus, um eine Patientenverfügung zu erschüttern. Zeigt aber etwa ein Demenzpatient innerhalb seiner Möglichkeiten noch aktive Lebensäußerungen oder gar Lebensfreude (vgl. Fall 2), kann

52 Ebenso Borasio/ Putz/Eisenmenger aaO. (Fn. 5).

53 Ebenso Paehler BtPrax 2003, 141, 143. 
dies die Schlussfolgerung tragen, er halte offenbar an seinem vormaligen Wunsch, nicht (mehr) behandelt zu werden, nicht mehr fest.

Die von der Bundesregierung eingesetzte Arbeitsgruppe »Patientenautonomie am Lebensende « unter Leitung von Vorsitzendem Richter am Bundesgerichtshof a. D. Klaus Kutzer hat in ihrem Bericht vom 10.06.2004 ${ }^{54}$ folgende Beispielsituationen für den Inhalt einer Patientenverfügung vorgeschlagen:

$»$ Wenn ich

- mich aller Wahrscheinlichkeit nach unabwendbar im unmittelbaren Sterbeprozess befinde,

- mich im Endstadium einer unheilbaren, tödlich verlaufenden Krankheit befinde, selbst wenn der Todeszeitpunkt noch nicht absehbar ist...,

- in Folge einer Gehirnschädigung meine Fähigkeit, Einsichten zu gewinnen, Entscheidungen zu treffen und mit anderen Menschen in Kontakt zu treten, nach Einschätzung zweier erfahrener Ärztinnen oder Ärzte (können namentlich benannt werden) aller Wahrscheinlichkeit nach unwiederbringlich erloschen ist, selbst wenn der Todeszeitpunkt noch nicht absehbar ist. Dies gilt für direkte Gehirnschädigung z.B. durch Unfall, Schlaganfall oder Entzündung ebenso wie für indirekte Gehirnschädigung z.B. nach Wiederbelebung, Schock oder Lungenversagen. Es ist mir bewusst, dass in solchen Situationen die Fähigkeit zu Empfindungen erhalten sein kann und dass ein Aufwachen aus diesem Zustand nicht ganz sicher auszuschließen, aber unwahrscheinlich ist,

- in Folge eines weit fortgeschrittenen Hirnabbauprozesses (z.B. bei Demenzerkrankung) auch mit ausdauernder Hilfestellung nicht mehr in der Lage bin, Nahrung und Flüssigkeit auf natürliche Weise zu mir zu nehmen.

- Eigene Beschreibung der Anwendungssituation: [Anmerkung: Es sollten nur Situationen beschrieben werden, die mit einer Einwilligungsunfähigkeit einhergehen können.] « Diesen Situationsbeispielen zur Übernahme in eine Patientenverfügung kann im Sinne dieses Beitrages uneingeschränkt zugestimmt werden. Diesbezüglichen Wünschen auf Behandlungsabbruch wird in aller Regel entsprochen werden können und müssen.

54 Im Internet abgelegt unter www.bmj.de/media/archive/695.pdf. 